\title{
Hox Genes Regulate Muscle Founder Cell Pattern Autonomously and Regulate Morphogenesis Through Motor Neurons
}

\author{
Devkanya Dutta ${ }^{1}$, M. Umashankar ${ }^{1}$,Edward. B. Lewis ${ }^{2,8}$, Veronica Rodrigues ${ }^{1,3}$ and K. VijayRaghavan ${ }^{1}$ \\ ${ }^{1}$ National Centre for Biological Sciences, Tata Institute of Technology, Pasadena, California, USA \\ ${ }^{2}$ Division of Biology, California Institute of Technology, Pasadena, California, USA \\ ${ }^{3}$ Department of Biological Sciences, Tata Institute of Fundamental Research, Mumbai, India
}

\begin{abstract}
The differentiation of myoblasts to form functional muscle fibers is a consequence of interactions between the mesoderm and ectoderm. The authors examine the role of segment identity in directing these interactions by studying the role of Hox genes in patterning adult muscles in Drosophila. Using the 'four-winged fly' to remove Ultrabithorax function in the developing adult, the authors alter the identity of the ectoderm of the third thoracic segment towards the second and show that this is sufficient to inductively alter most properties of the mesoderm - myoblast number, molecular diversity, and migration pattern - to that of the second thoracic segment. Not all aspects of myogenesis are determined by the segment identity of the ectoderm. The autonomous identity of the mesoderm is important for choosing muscle founder cells in the correct segmental pattern. The authors show this by removal of the function of Antennapedia, the Hox gene expressed in the mesoderm of the third thoracic segment. This results in the transformation of founder cells to a second-thoracic pattern. The authors also report a role for the nervous system in later aspects of muscle morphogenesis by specifically altering Ultrabithorax gene expression in motor neurons. Thus, ectoderm and mesoderm segment identities collaborate to direct muscle differentiation by affecting distinct aspects of the process.
\end{abstract}

Keywords: Antennapedia, dumbfounded, Hox, motor neurons, muscle, myogenesis, Ultrabithorax

\section{INTRODUCTION}

Myogenesis in the fruitfly, Drosophila melanogaster, is illustrative of how the final morphogenesis of a tissue is the cumulative effect of 'canalization' (Waddington \& Robertson, 1966) during development (Rochlin et al., 2009; Dutta \& VijayRaghavan, 2006; Roy \& VijayRaghavan, 1999). In making the adult thoracic musculature, mesodermal cells are set aside in a segment-specific pattern in the embryo as progenitors of adult muscle (Bate et al., 1991). Later, adult myoblasts in the second thoracic segment associate with the progenitors of the dorsal or ventral thoracic epidermis, the wing and leg imaginal discs (Lawrence, 1982). Wing disc myoblasts are further divided to contribute to the formation of the direct and indirect flight muscles (Lawrence, 1982; Ghazi et al., 2000). Next, during metamorphosis, myoblasts migrate to specific epidermal sites where founder myoblasts are chosen to seed the formation of muscle fibers (Fernandes et al., 1991; Dutta et al., 2004). Developmentalgenetics approaches have been used to demonstrate how this progressive narrowing of cellular options can be seen as a consequence of a combination of inductive signals and cell-autonomous, lineage-dependent decisions (Fernandes et al, 1994; Roy et al 1997; Roy \& VijayRaghavan, 1997).

These sequential decisions can be visualized as the localized expression, and requirement, of genes during development. Thus, for example, all adult myoblasts express Twist (Twi) (Bate et al., 1991; Currie \& Bate, 1991). All wing disc-associated myoblasts express $\mathrm{Cut}(\mathrm{Ct})$, but those that will contribute to the indirect flight muscles also express Vestigial (Vg) (Sudarsan et al., 2001) and, later on, Erect wing (Ewg) (DeSimone et al., 1995). Those that contribute to the direct flight muscles express Apterous (Ghazi et al., 2000). Vg expression is maintained by Wingless signaling from the epidermis (Sudarsan et al., 2001). The number of fibers and their location is determined by the choice of dumbfounded-expressing founder cells, special myoblasts chosen from amongst their neighbors (Dutta et al., 2004), whereas fusion-competent myoblasts, expressing cell-surface molecules encoded by genes such

Received 3 February 2010; Accepted 16 May 2010.

${ }^{\S}$ Deceased.

Address correspondence to Veronica Rodrigues or K. VijayRaghavan, National Centre for Biological Sciences, Tata Institute of Fundamental Research, GKVK, Bellary Road, Bangalore, 560065, India. E-mail: veronica@ ncbs.res.in; vijay@ncbs.res.in 
as sticks and stones (Bour et al., 2004), are attracted to founder cells to fuse and form a multinucleate fiber. Differentiating fibers search and attach to stripe-expressing epidermal attachment sites and are innervated by motor neurons in a pattern recognizable and characteristic of each muscle (Fernandes et al., 1996; Fernandes \& VijayRaghavan, 1993).

The pattern of muscles can vary dramatically between segments and here too the indirect flight muscles (IFMs) are illustrative. IFMs are composed of two types of muscles-the dorsolongitudinal muscles (DLMs) and the dorsoventral muscles (DVMs) (Fernandes et al., 1991). How is the thorax-specific pattern of IFMs generated? What are the relative roles of the ectoderm and mesoderm in generating this pattern? The first studies that addressed the above questions did so by examining the phenotype of muscles in the adult using polarized-light microscopy (Egger et al., 1990). Subsequently, the use of antibodies and reporter genes, which labeled myoblasts and differentiating muscles, allowed analysis during embryonic and pupal development (Roy et al., 1997; Roy \& VijayRaghvan, 1997; Fernandes et al., 1994).

The results from these studies have been summarized in a review on adult Drosophila myogenesis (Dutta \& VijayRaghavan, 2006). Briefly, the Hox identity of the dorsal mesothoracic mesoderm is characterized by the absence of expression of the genes of the Antennapedia (Antp) and bithorax complexes (Roy et al., 1997). This is an obligate requirement, and the misexpression of Hox genes abolishes the pattern of IFMs (Roy et al., 1997). The segment identity of the wing disc ectoderm-itself not expressing any Hox gene-dictates the proliferation, spatial distribution pattern on the disc, and migration of myoblasts. Yet, although all these aspects are transformed from a T3 to a T2 pattern in the four-winged fly (generated by combinations of Ultrabithorax alleles) (Fernandes et al., 1994), the transformed T3 has essentially no indirect flight muscles (Fernandes et al., 1994; Egger et al., 1990; Ferrus \& Kankel, 1981). The likely explanation for this is that segment-specific muscle differentiation requires the action of both inductive signals and autonomous cues. These autonomous cues, it has been inferred, could well be the segment identity of founder cells (Roy \& VijayRaghvan, 1997).

The more recent availability of additional markers of IFM development, namely the proteins $\mathrm{Vg}$ and $\mathrm{Ct}$ (which impart specific identity to subsets of myoblasts) (Sudarsan et al., 2001) and the reporter lacZ line dumbfounded (duf)-lacZ (which labels adult muscle founder cells) (Dutta et al., 2004, 2005), and their functional characterization allows the above inference to be directly tested. Specifically, we examined the expression of these markers in the mesoderm of the homeotically transformed T3 in four-winged mutants. We examined the expression of $\mathrm{Vg}$ and $\mathrm{Ct}$ in previously analyzed allelic combinations of mutation in the bithorax complex that give the four-winged phenotype (Fernandes et al., 1994) and, additionally, in new combinations that show a greater expressivity of the phenotype. We also examined the expression pattern of the muscle founder cell marker $d u f$-lac $Z$ in these genetic contexts. Results from these studies were consistent with our hypothesis: all aspects of the mesoderm, except the founder cell pattern for the IFMs, were transformed in the four-winged fly. The hypothesis - that founder cell segmental identity is specified autonomously-predicts that the removal of Antp function in the T3 mesoderm of the four-winged fly should lead to a T2 founder pattern. We show that this indeed is the case. With the selection of T2 founders in T3, and all properties of T3 myoblasts already transformed to a $\mathrm{T} 2$ pattern, the additional prediction would be that this would lead also to the development and differentiation, in T3, of muscles that resemble the IFMs. This too is the case. We know that inductive signal(s) are important for the selection of founder cells (Dutta et al., 2005). Our studies therefore make the point that the autonomous segment identity of myoblasts is critical for the correct segment-specific response to such inductive signals. Once muscles are patterned by the choice of founder cells, morphogenesis begins and requires interactions with the environment: with tendon cells and the nervous system for example. We examined the role of Hox gene requirement in the nervous system on muscle morphogenesis, by reducing $U b x$ expression in the motor neurons of $\mathrm{T} 3$ and by misexpression of $U b x$ in the motor neurons of T2. Our results point to an important role for the nervous system in the later aspects of muscle morphogenesis and maintenance.

\section{MATERIALS AND METHODS}

\section{Fly Strains}

Given below are the genotypes of the three types of fourwinged flies used in this study.

(a) Triple mutant-abx $b x^{3} p b x / \operatorname{In}(3 L) P$ ss $a b x b x^{3} p b x$. anterobithorax $(a b x)$, bithorax $\left(b x^{3}\right)$, and postbithorax $(p b x)$ are regulatory mutations of Ultrabithorax (Lewis, 1978). In(3L)P (Inversion(3L)Payne) is an inversion whose breakpoints include 63B8-9 and 72E1-2. Although the triple-mutant combination shows a $100 \%$ penetrance, the expressivity of the phenotype is not quite complete, presumably because the regulatory regions affected by each mutation are not completely inactivated.

(b) Quad/triple mutant - abx bx $\mathrm{bx}^{3} U b x^{61 d} p b x / a b x b x^{3} p b x$. $U b x^{61 D}$ is a weak mutation in the $U b x$ coding region (Lewis, 1980). In quad/triple mutant combination, $U b x$ is inactivated to a greater extent than triple 
mutants, as manifested by a higher expressivity of the four-winged phenotype.

(c) Antp-triple mutant-Antp ${ }^{1}$ red $^{1} \operatorname{In}(3 L) P$ ss $a b x b x^{3}$ $p b x / D f(3 R) A n t p^{6}, A n t p^{6}$ red $a b x, b x^{3} p b x$.

$A n t p^{6}$ and $A n t p^{l}$ are hypomorphic mutations in Antennapedia

All homeotic stocks, carrying the different allelic combinations, were maintained over TM6, $\mathrm{Hu}$, Tb balancer. Four-winged mutants were generated by crossing the appropriate stocks and selecting non-Tb pupae. dumbfounded (duf)-lacZ, an enhancer trap line having a Pelement nuclear-localizing lacZ inserted within the promoter region of $d u f$ (Ruiz-Gomez et al., 2000; Nose et al., 1998), was crossed into the background of the Hox mutant stocks using standard genetic techniques. Four-winged mutants were generated such that they contained at least one copy of the $d u f$-lac $Z$ transgene.

The 'motor neuron' GAL4 strain OK371-GAL4 (Mahr \& Aberle, 2006) was used to drive expression from the UAS-Ubx-RNAi transgene or the UAS-Ubx transgene. OK371-GAL4 is a P-element insertion in the vesicular glutamate transporter locus and is expressed in adult motor neuron precursors and in mature motor neurons in addition to other glutamatergic neurons (Mahr and Aberle, 2006; Brierley et al., 2009). The P103.3-GAL4 (Consoulas et al., 2002) is expressed in the MN5 motor neuron, which innervates the dorsal longitudinal muscles and was used to misexpress Ubx in this motor neuron. The consequences on muscles of the T3 leg or the IFMs were examined in polarized light preparations as described below.

\section{Tissue Preparation}

White prepupae were collected on moist filter paper in a Petri dish and grown at $25^{\circ} \mathrm{C}$ for the requisite interval. $\mathrm{Pu}-$ pal tissues and imaginal discs were prepared for immunohistochemistry (described previously in Fernandes et al., 1991) and were mounted in $70 \%$ glycerol, for X-galstained preparations, or in Vectashield mounting medium (Vector Labs, Burlingame, CA), for fluorescence-labeled preparations. Adult thoracic preparations were prepared by anesthetizing the flies (or taking out the pharate adults from pupal cases), sticking one at a time on a slide using a drop of $70 \%$ glycerol and then freezing in liquid nitrogen. They were then cut sagitally along the center, dehydrated through an ethanol series, mounted in Canada balsam, and viewed under polarized light.

\section{Immunohistochemistry}

Mouse monoclonal anti-Antp antibody, a gift from Danny Brower (University of Arizona, Tucson, USA), was used at a dilution of 1:200. Monoclonal antibodies anti-Cut and
22C10 (both obtained from Developmental Studies Hybridoma Bank) were used at a dilution of 1:50. Rabbit anti$\mathrm{Vg}$ antibody, a gift from Sean Carroll (University of Wisconsin, Madison, USA), was used at a dilution of 1:200. Rabbit anti-myosin heavy chain (MHC) antibody, a gift from Dan Kiehart (Duke University, Durham, USA), was used at a dilution of 1: 500. Rabbit anti- $\beta$-galactosidase antibody (Molecular Probes, Eugene, OR) was used at a dilution of 1:5000. Rabbit anti-Twist antibody, gifted by Siegfried Roth (University of Cologne, Cologne, Germany), was used at a dilution of 1:5000. For assaying $\beta$-galactosidase activity of $d u f$-lac $Z$ in different mutants, dissected pupae were stained overnight with chromogenic substrate X-gal (Sigma, St. Louis, Missouri, USA). Secondary antibodies conjugated to Alexa Fluor dyes (Molecular Probes) were used at a dilution of 1:200-Alexa 488 for green labeling and Alexa 568 for red labeling.

\section{Microscopy}

Differential interference contrast (DIC) and fluorescent images of pupal preparations and polarized images of hemithoracic preparations were taken using a Nikon Eclipse E1000 microscope. Fluorescent preparations of imaginal discs were scanned in the confocal microscope (MRC-1024; Bio-Rad Laboratories, Hercules, California, USA) and images were analyzed using the software Metamorph (version 4.5) (Universal Imaging).

\section{RESULTS}

\section{Vestigial and Cut Expression in T3 Myoblasts Is Transformed to a T2 Pattern in Triple-Mutant Larva}

Vestigial ( $\mathrm{Vg}$ ) and $\mathrm{Cut}(\mathrm{Ct})$ expression divide wing discassociated myoblasts into groups that adopt two different fates during pupal myogenesis-one group expresses $\mathrm{Vg}$ and $\mathrm{Ct}$ (at low levels) whereas the other group expresses Ct (at higher levels) but not Vg (Sudarsan et al., 2001) (Figure 1A-C). The IFMs develop from the $\mathrm{Vg}-\mathrm{Ct}-$ expressing subgroup of myoblasts (Sudarsan et al., 2001). The haltere disc-associated myoblasts of the adjacent T3 segment also express Vg and Ct (Figure 1D-F). However, unlike the wing disc-associated myoblasts, $\mathrm{Vg}$ and $\mathrm{Ct}$ are expressed at comparable levels in all haltere discassociated myoblasts.

In triple-mutant four-winged flies, which are homozygous for three mutant alleles of the Ultrabithorax gene (genotype given in Materials and Methods), the T3 ectoderm is transformed to a T2 identity (Lewis, 1978). But the myoblasts associated with the homeotically transformed T3 (HT3) discs maintain their T3 identity and continue to express Antp (which is expressed in wild-type 
A

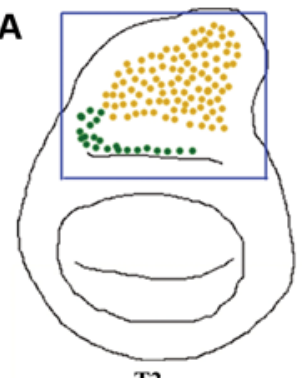

E

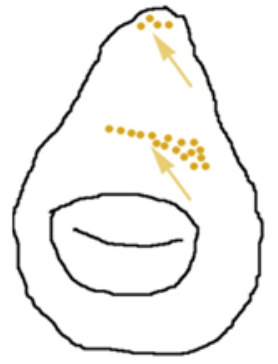

T3

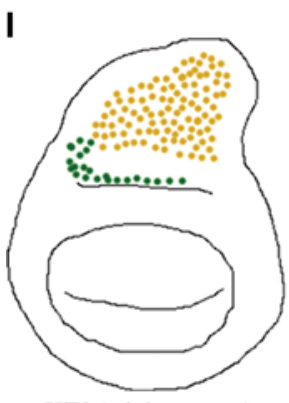

HT3 (triple mutant)
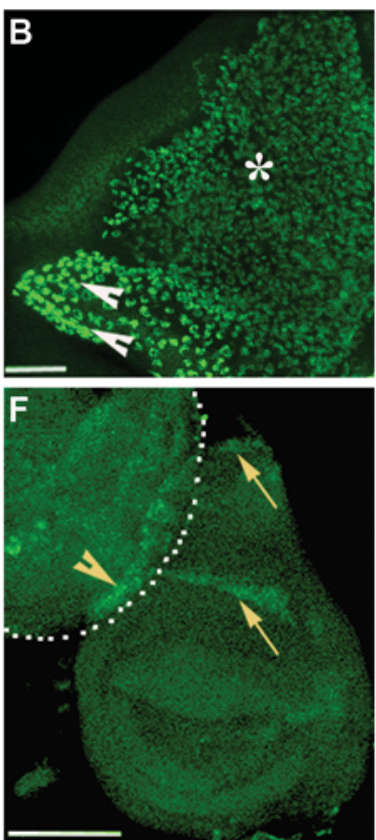

J

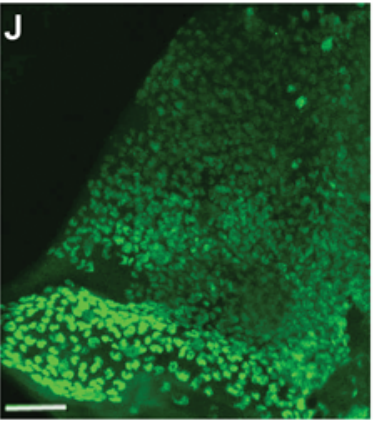

Cut
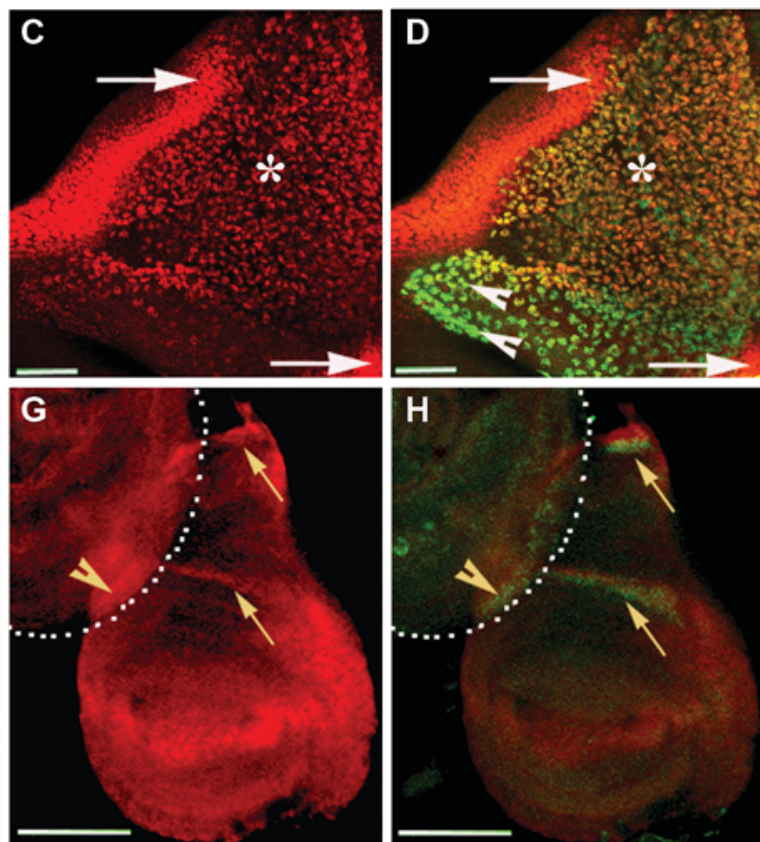

K

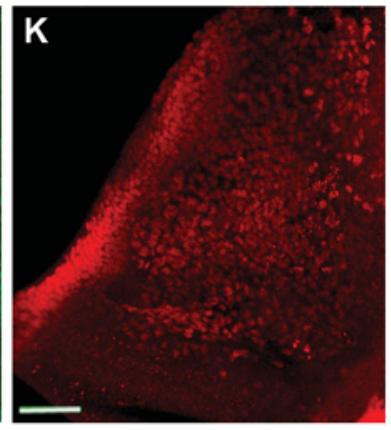

Vestigial
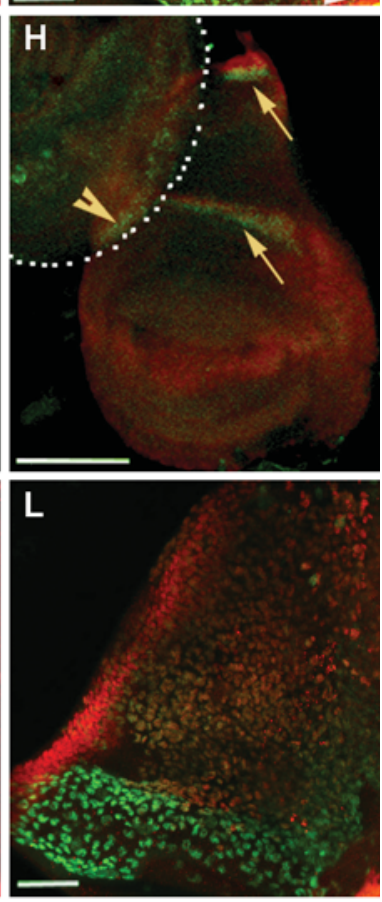

Merged

Figure 1. Expression of $\mathrm{Vg}$ and $\mathrm{Ct}$ in dorsal myoblasts of wild-type and triple-mutant larvae. (A) Diagram of a wing disc showing the myoblasts overlying the epithelium of the notum. Myoblasts expressing Vg and low levels of Ct are shown as yellow dots, whereas myoblasts expressing higher levels of $\mathrm{Ct}$ are shown as green dots. Blue box shows the approximate region that has been magnified in $\mathbf{B}, \mathbf{C}$, and $\mathbf{D}$. (B, C, D) Magnified images of the presumptive notal region of wild-type third instar larval wing disc (area outlined in $\mathbf{A}$ ) double-labeled with $\mathrm{Ct}(\mathbf{B})$ and $\mathrm{Vg}(\mathbf{C})$. Merged image is D. Proximal cells (white asterisk) express both $\mathrm{Vg}$ and $\mathrm{Ct}$ (in lower levels). Distal cells (white arrowheads in $\mathbf{B}$ and $\mathbf{D}$ ) lack Vg but express higher levels of Ct. White arrows in $\mathbf{C}$ and $\mathbf{D}$ show Vg staining in the epidermal cells of the wing disc. (E) Diagram of a haltere disc showing the spatial distribution of myoblasts associated with it. Yellow dots represent myoblasts, all of which express $\mathrm{Ct}$ and $\mathrm{Vg}$. (F, G, H) Haltere disc of a wild-type third instar larva stained with $\mathrm{Ct}(\mathbf{F})$ and $\mathrm{Vg}(\mathbf{G})$. Merged image is shown in $\mathbf{H}$. In this preparation, haltere disc is attached to a T3 leg disc. The white dotted curve demarcates the leg disc from the haltere disc. In comparison to the wing disc, haltere disc has fewer myoblasts. These myoblasts are located in two distinct clusters (yellow arrows) — a smaller cluster present at the proximal region and a larger cluster present in the distal region. All myoblasts express both $\mathrm{Ct}$ and $\mathrm{Vg}$. Yellow arrowheads indicate the myoblasts that are associated with the leg disc. (I) Diagram of a HT3 disc of a triple-mutant larva showing patterns of spatial distribution and molecular diversity of myoblasts identical to those of wild-type T2. (J, K, L) HT3 disc of a triple-mutant larva showing expression of $\mathrm{Ct}(\mathbf{J})$ and $\mathrm{Vg}(\mathbf{K})$. $\mathbf{L}$ is the merged image. Expression patterns of $\mathrm{Ct}$ and $\mathrm{Vg}$ in HT3 myoblasts are identical to that of wild-type T2 disc-associated myoblasts (shown in $\mathbf{B}, \mathbf{C}$, and $\mathbf{D}$ ). Scale bar $=30 \mu \mathrm{m}$ (B-D, J-L), $50 \mu \mathrm{m}$ (F-H).

T3 myoblasts) (Roy et al., 1997). Despite the presence of Antp, these myoblasts are transformed to a T2 pattern with respect to their number, spatial distribution, and migration pattern (Fernandes et al., 1994; Roy et al., 1997; Roy \& VijayRaghavan, 1997). We examined whether $\mathrm{Vg}$ and $\mathrm{Ct}$ expression patterns are also transformed in HT3 myoblasts of triple mutants. Figure 1J and $\mathrm{K}$ show $\mathrm{Ct}$ and $\mathrm{Vg}$ expressions, respectively, in
HT3 myoblasts of triple-mutant larvae. The expression patterns of the two proteins were found to be identical to that observed in wild-type T2 myoblasts (as shown in Figure $1 \mathrm{~B}$ and $\mathrm{C}$ ). This shows that generation of molecular diversity, imparted by $\mathrm{Vg}$ and $\mathrm{Ct}$ proteins, is not dependent on the homeotic address of the myoblasts and is governed entirely by the segmental identity of the ectoderm. 


\section{Founders for the IFMs Are Absent in HT3 of Triple-Mutant Pupae}

A founder cell seeds the formation of a muscle fiber, and an appropriate number of such founders generate multifiber adult muscles at the correct position (Dutta et al., 2004, 2005). Thus, for DVMs, a set of three, two, and two $d u$ f-lacZ-expressing cells, corresponding to DVM I, II, and III, respectively, are selected at the correct location relative to the epidermis. Figure $2 \mathrm{~B}$ and $\mathrm{F}$ show the $d u f$-lacZ-expressing founder cells of DVM I (long white arrows) and DVM II (short white arrows). The DLMs, in contrast to the DVMs (and most other adult muscles), develop by a 'template-dependent' mechanism: a set of larval muscle fibers or 'templates' escape histolysis at the onset of metamorphosis and serve as scaffolds for DLM fiber formation (Fernandes et al., 1991). The larval templates have all the characteristics of founders for the DLMs (Farrell et al., 1996; Fernandes \& Keshishian, 1996; Dutta et al., 2004). They also express the founder marker duf-lacZ (shown by asterisks in Figure 2B) (Dutta et al., 2004). The DVM founders and the larval templates, collectively, will henceforth be referred to as IFM founders.

We examined the expression of duf-lacZ in the T3 of wild-type pupa. A cluster of $\sim 14$ duf-lacZexpressing myoblasts are observed in T3 (Figure 2J, $\mathrm{L}$; demarcated with dotted lines in Figure 2B-D). The
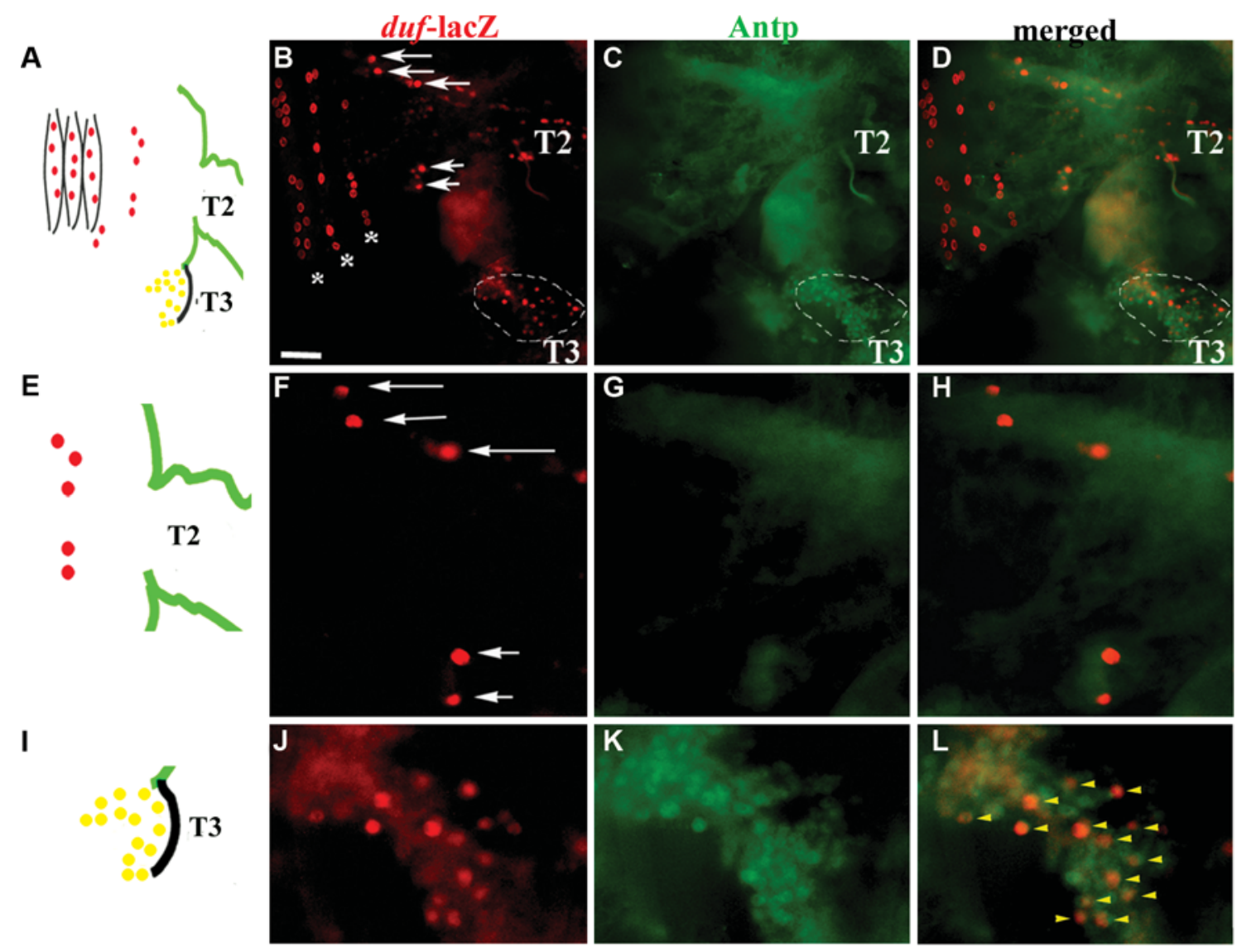

Figure 2. Wild-type pattern of founder cells in T2 and T3 segments. Twelve-hour APF duf-lacZ pupa is double-labeled with anti$\beta$-Galactosidase (red) and anti-Antp (green). (A, E, I) Diagrams of flat pupal preparations showing the spatial location of the duflacZ-expressing founder cells in the T2 and T3 mesoderm. A show both T2 and T3 segments whereas $\mathbf{E}$ and I show magnified versions of ventral regions of T2 and T3, respectively. duf-lacZ-expressing larval templates are shown as red dots within black brackets (in A), whereas DVM founders are depicted by red dots (in A, E). duf-lacZ-expressing founder cells of T3, which also express Antp, are indicated by yellow dots (in A, I). (B) duf-lacZ pupal preparation showing founder cells for DVM I (long arrows), DVM II (short arrows), and the DLM templates (white asterisks) in T2. Dotted region encompasses the area where the founder cells of T3 are present. (C) Same preparation as in B, showing expression of Antp. In T2, Antp is expressed in the ectoderm but not in the myoblasts. In T3, Antp is expressed in myoblasts (demarcated by the dotted region). (D) Merged image of $\mathbf{B}$ and $\mathbf{C}$. (F-H) Magnified view of the same pupal preparation in $\mathbf{B}$, showing the region where DVM founders are present. (F) duf-lacZ-expressing founders of DVM I (long arrows) and of DVM II (short arrows). (G) Antp expression in the ectoderm. (H) Merged image of $\mathbf{F}$ and $\mathbf{G}$. (J-L) Magnified view of the dotted region in B. (J) duf-lacZ-expressing cells in T3. (K) Antp expression in all T3 myoblasts. (L) Merged image of $\mathbf{J}$ and $\mathbf{K}$, showing the subset of T3 cells that are also expressing duf-lacZ. These cells are the founder cells of T3. Anterior: top; dorsal midline: left. Scale bar $=23 \mu \mathrm{m}(\mathbf{B}-\mathbf{D})$. 
spatial pattern of the $d u f-l a c Z$ founder cells in T3 is distinctly different from those in T2. T3 founders are present in a position more ventrally that those of the DVMs in T3. Also, no founder cells are present in the dorsal region of $\mathrm{T} 3$.

We next examined whether founder cells for IFMs are present in HT3 of triple-mutant pupae. We did not detect ectopic DLM templates or DVM founders in triple-mutant HT3 (number of pupae scored $=80$ ) (Figure 3 ). Correspondingly, we also did not observe any ectopic IFMs

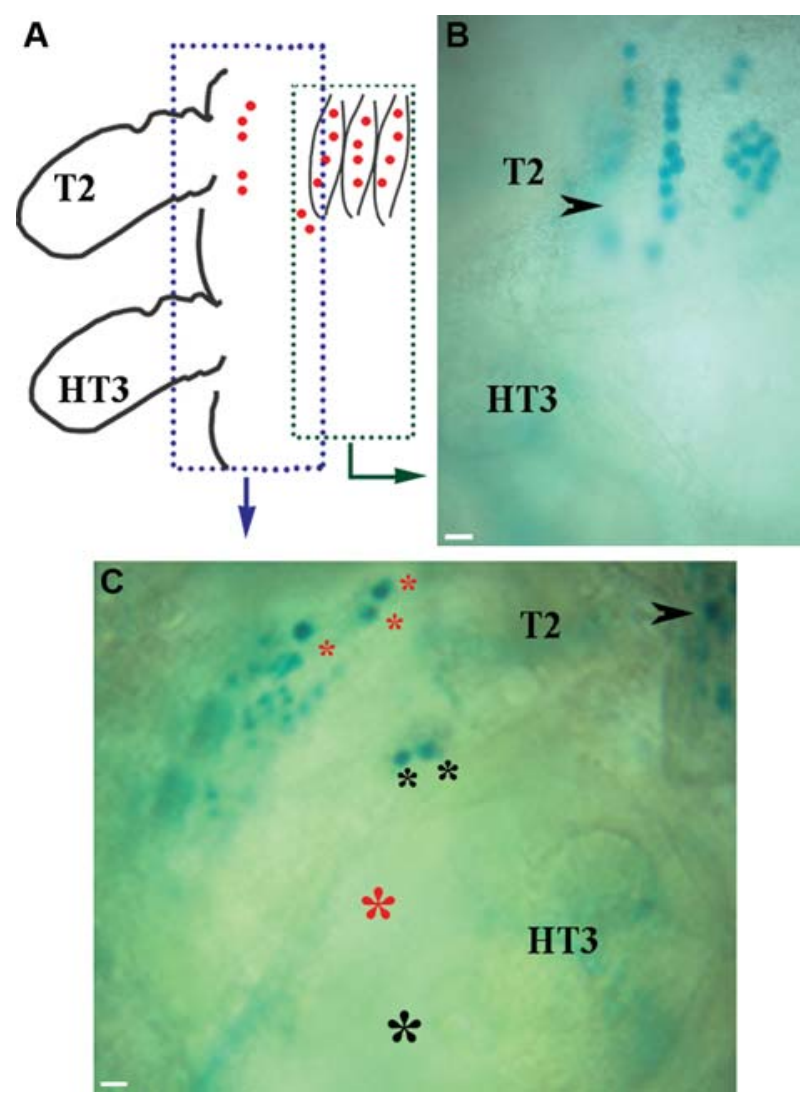

Figure 3. Pattern of duf-lacZ-expressing founders in triplemutant pupa. (A) Diagram of a flat preparation of triple-mutant pupa showing DVM founders (red dots) and larval templates (red dots enclosed in black brackets) in T2. The green and blue dotted boxes outline the areas of the preparation that are in view in $\mathbf{B}$ and $\mathbf{C}$, respectively. (B) A 12-h APF pupa of the genotype $d u f$ lacZ/+: In (3L) P ss $a b x b x^{3} p b x / a b x b x^{3} p b x$ (triple mutant), assayed for $\beta$-galactosidase activity. The three larval templates, expressing duf-lacZ, are present in T2. The black arrowhead indicates the template (partially out of focus) that is farthest from the dorsal midline. No templates are present in the corresponding region of HT3. (C) Same pupal preparation as in $\mathbf{B}$ but the region shown here is further away from the dorsal midline than in $\mathbf{B}$. The black arrowhead indicates the template that is farthest from the dorsal midline (same template indicated in $\mathbf{B}$ ). The founder cells of DVM I (red, small asterisks) and DVM II (black, small asterisks) are present in T2. The DVM III founders are out of focus in this preparation. In the equivalent regions of HT3 (indicated by bold asterisks), no founders are present. Anterior: top; dorsal midline: right. Scale bar $=10 \mu \mathrm{m}$. in the HT3 thorax of adult triple-mutant flies (number of flies screened $=100$ ) (data not shown; also reported in Fernandes et al., 1994; Egger et al., 1990; Ferrus \& Kankel; 1981). Thus, the two events critical for generating the fibers for IFMs - selection of DVM founders and the persistence of larval templates-fail to occur in HT3 of triple mutants, suggesting that this could be the cause of absence of IFMs in the transformed T3 segment.

\section{IFM Founders Are Absent in HT3 Segment of Quadruple/Triple Mutants}

It is possible that the combination of the three regulatory $U b x$ mutations in triple-mutant flies is not effective for a complete transformation of T3 ectoderm towards a T2 identity. If selection of founder cells in a segment is governed entirely by its ectodermal identity, then a more extreme inactivation of $U b x$ than that occurs in the triple mutant might lead to selection of founders in HT3. For a stronger ectodermal transformation, we generated fourwinged flies having an additional $U b x$ mutation ( $U b x^{6 l d}$ ) in the triple-mutant background. These flies, referred to as quadruple/triple or quad/triple (genotype given in Materials and Methods), had a higher expressivity of the fourwinged phenotype than in triple-mutant flies. Like the triple mutants, pupae of the quad/triple genotype did not show the presence of any DVM founders or larval templates in their HT3 segment (data not shown) (number of pupae scored $=70$ ). Adult flies of quad/triple genotype did not have ectopic IFMs in the HT3 (number of flies screened $=100)($ data not shown).

\section{IFM Founders Are Present in HT3 of Antp-Triple Mutant Flies}

As mentioned earlier, although the transformation of T3 ectoderm to a T2 identity takes place in the triple mutants, all myoblasts of HT3 retain their T3 identity, as seen by their expression of Antp (Roy et al., 1997). This is also the case in the quad/triple-mutant combination (data not shown). We hypothesized that the absence of IFM founders in the HT3 of the triple and quad/triple mutants is due to the mesoderm retaining a T3 identity as seen by the expression of Antp in HT3 myoblasts. If this is true, then, removing Antp completely or decreasing its level considerably should allow IFM founders to be selected in HT3. To reduce Antp levels in HT3, we introduced hypomorphic mutations of Antp in the triple-mutant background using standard recombination procedure. The four-winged flies, generated by these mutants, were referred to as Antptriple mutants.

To confirm decreased levels of Antp in HT3 of Antptriple mutants, we immunostained HT3 discs of triple-mutant 
and Antp-triple mutant larvae with anti-Antp and anti-Vg (to mark the myoblasts) (Figure 4). Compared to the triple mutant (Figure 4B), HT3 myoblasts of the Antp-triple mutants (Figure 4D) showed considerable decrease in levels of Antp. A decrease in Antp levels should not affect the $\mathrm{Vg}-\mathrm{Ct}$ allocation of the myoblasts, since this property is governed entirely by ectodermal identity. As expected, Vg-Ct expression pattern in HT3 myoblasts of both of these mutants were found to be identical to the T2 pattern (data not shown). In addition to the strong expression of Antp in HT3 myoblasts of triple-mutant larvae, Antp expression was also observed in the HT3 ectoderm of triple mutants (Figure 4B). This ectodermal expression was not observed in the HT3 of Antp-triple mutants (Figure 4D). The absence of ectodermal Antp did not produce any phenotypic changes in the HT3 ectoderm of the Antp-triple mutant pharate adults (data not shown).

We examined the expression of $d u f$-lacZ in Antp-triple mutant pupae. We observe larval muscles that have escaped histolysis and are present at a location corresponding to DLM templates, in the HT3 of these pupae. Figure 5B shows a $12 \mathrm{~h}$ APF (after puparium formation) pupa having three template-like fibers in its HT3. Some pupae had two, instead of three, template-like fibers in their HT3. We also observed clusters of three and two duf-lacZ-expressing cells in HT3, at positions analogous to that occupied by founders of DVM I and DVM II, respectively, in wild-type T2. Figure 5C shows two duf-lacZ-expressing cells of HT3 (white asterisks) at a position similar to that occupied by DVM II founders in T2. Based on the observations that the number of founders in a cluster matches with that for wild-type DVMs and that in wild-type T3 no duf-lacZ-expressing cells are present in equivalent positions (Figure 2B, D), we would like to suggest that these cells are the ectopic founder cells for DVMs. A total number of 50 Antp-triple mutant pupae were dissected and examined for expression of DLM and DVM founder cell markers in HT3. Ten animals showed ectopic founders, of which five showed both larval templates and DVM founders, three showed only larval templates, and two showed only DVM founders. Staining with anti-MHC antibody at a developmentally later stage (32 h APF) of Antp-triple mutant
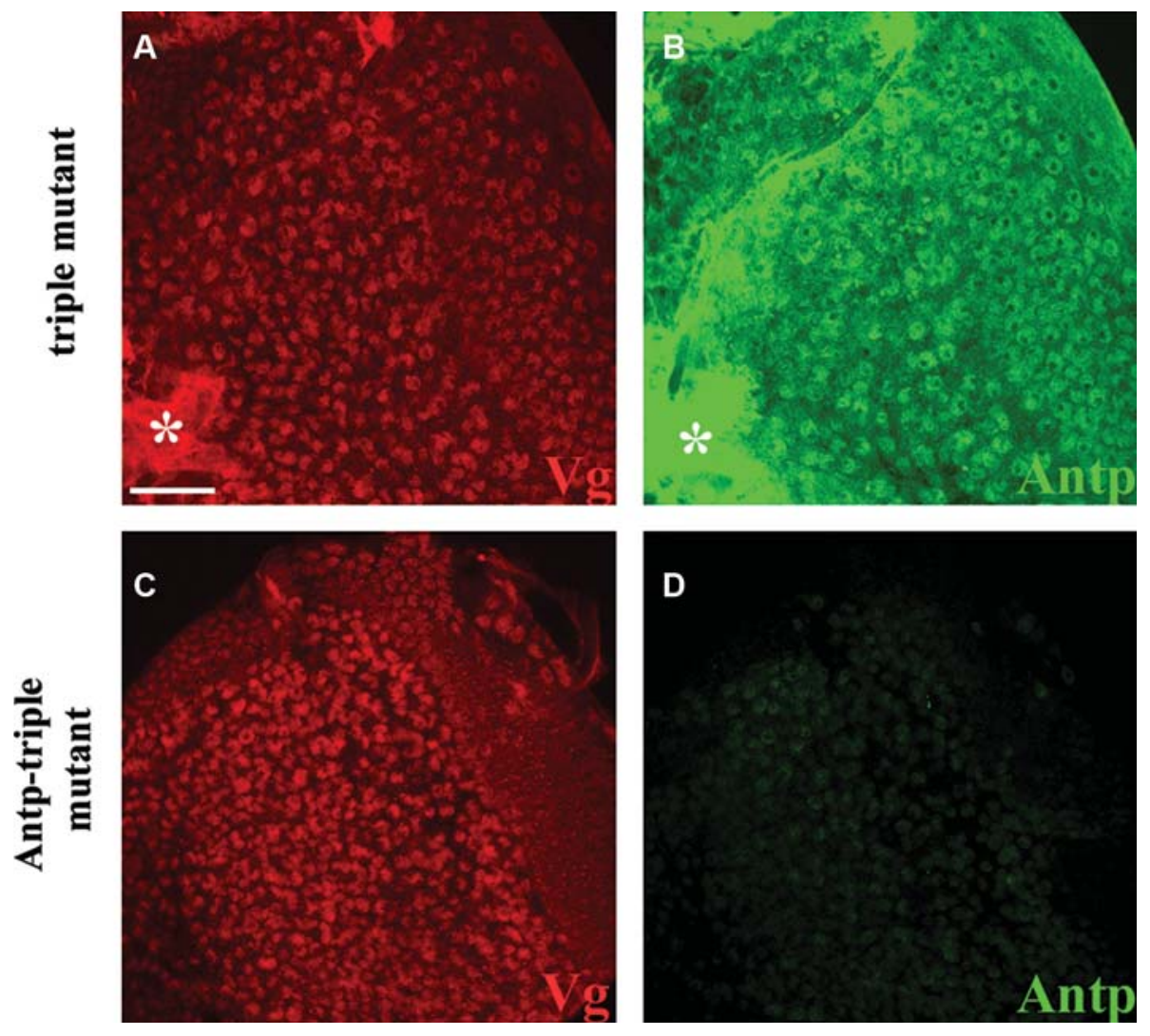

Figure 4. Decreased level of Antp in HT3 mesoderm of Antp-triple mutants. HT3 wing discs labeled with anti-Vg (red) and anti-Antp (green) to compare the levels of Antp protein in myoblasts. All preparations were treated identically during antibody staining and imaged under identical conditions. (A, B) Presumptive notal region of the HT3 disc of a triple-mutant larva, showing Vg-expressing myoblasts (A) that also expresss Antp (B). The bright fluorescent patch (indicated by white asterisk in $\mathbf{A}$ and $\mathbf{B}$ ) is due to tissue debris sticking to the disc. (C, D) Presumptive notal region of the HT3 wing disc of Antp-triple mutant larva showing expression of Vg (C) and Antp (D). Level of Antp protein is significantly reduced in myoblasts of Antp-triple mutants (in D) compared to that in triple mutants (in B). HT3 mesoderm of Antp-triple mutant, thereby, resembles the wild-type T2 mesoderm in terms of Hox expression. Scale bar $=50 \mu \mathrm{m}$. 


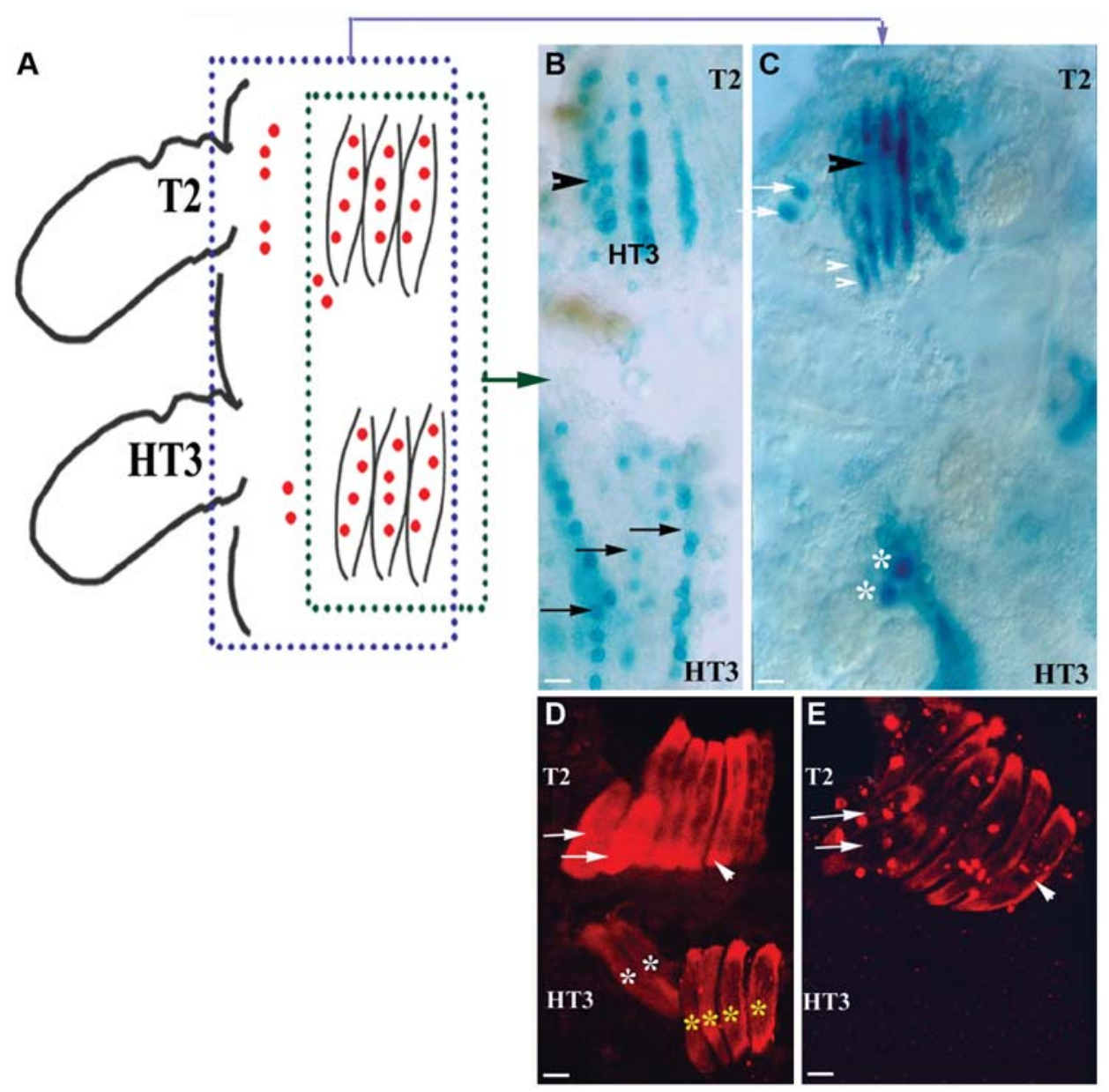

Figure 5. Ectopic IFM founders in HT3 of Antp-triple mutants. (A) Diagram of a flat preparation of Antp-triple mutant pupa. Red dots represent the DVM founders whereas red dots enclosed in black brackets represent the $d u f$-lacZ-expressing larval templates. Green- and blue-dotted boxes outline the approximate regions that are in view in $\mathbf{B}$ and $\mathbf{C}$, respectively. (B, $\mathbf{C}) \mathbf{X}$-gal staining, in two separate pupal

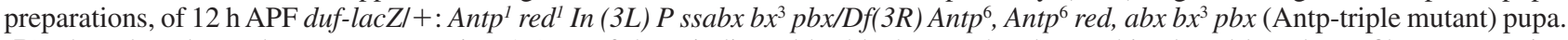
(B) Three larval templates are present in T2 (one of them indicated by black arrowhead). Nonhistolyzed larval myofibers expressing duf-lac $Z$ are present in the equivalent region in HT3 segment (black arrows), suggesting that these are transformed larval templates. (C) Founders for DVM II (white arrows), DVM III (white arrowheads), and larval templates (black arrowhead) are present in T2. Founders of DVM I are not in view. In HT3, two duf-lacZ-expressing cells (white asterisks) are present at a position that is analogous to that occupied by DVM II founders in T2. No template-like fibers are present in HT3 of this particular preparation. (D, E) Thirty-two hours APF preparations of Antp-triple mutant pupae stained with anti-MHC to observe the developing myotubes. (D) Six DLM fibers (white arrowhead) and DVM III fibers (white arrows) are visible in T2. In HT3, four DLM-like fibers (yellow asterisks) and two fibers of DVM II (white asterisks) are observed. (E) The DLMs (white arrowhead) and DVM III (white arrows) are present in normal pattern in T2. No ectopic IFM fibers are present in this preparation. In both $\mathbf{D}$ and $\mathbf{E}$, the normal DVM I and DVM II fibers in T2 are not included in the view of the image. Anterior: top; dorsal midline: right. Scale bar $=10 \mu \mathrm{m}(\mathbf{B}, \mathbf{C}), 25 \mu \mathrm{m}(\mathbf{D}, \mathbf{E})$.

pupae revealed the developing muscle fibers in their HT3. Figure 5D shows a pupal preparation that has DVM II fibers (white asterisks) and four DLM fibers (yellow asterisks) in its HT3 whereas Figure 5E shows a preparation that does not have any ectopic IFMs in HT3. Antp-triple mutant flies do not eclose from the pupal case. Pharate adults were scored for the presence of transformed IFMs (Figure 6). Fifteen out of 80 hemithoraces of Antp-triple mutant pharate adults showed IFMs in the HT3 (Figure $6 \mathrm{~F})$. When viewed under polarized light, these fibers did not show transverse striations, a morphological property manifested by tubular muscles but not fibrillar muscles like the IFMs (data not shown).

\section{A Role for the Nervous System in Muscle Morphogenesis, Revealed by Altering $U b x$ Expression in Motor Neurons}

As has been shown in several studies on the role of Hox genes in myogenesis (Fernandes et al., 1994; Roy et al., 1997; Sudarsan et al., 2001) and in this study, inductive 

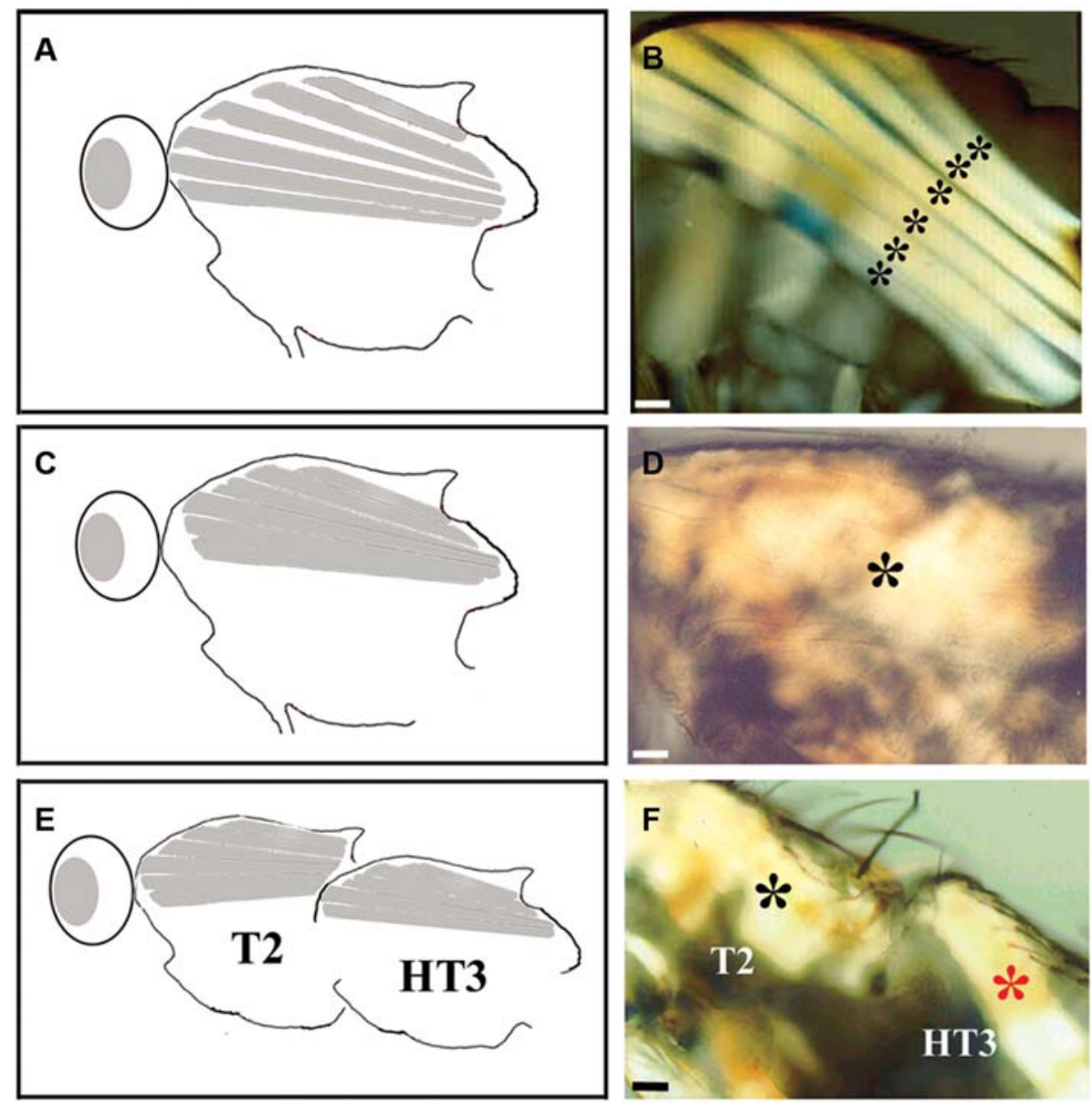

Figure 6. Ectopic IFM fibers in HT3 of Antp-triple mutant pharate adults. (A, C) Diagrammatic representation of the hemisegment of a wild-type adult fly (A) and a wild-type pharate adult fly $(\mathbf{C})$, showing the DLM fibers. (B) Hemithoracic preparation of a wild-type, normally enclosed, adult fly showing the six DLM fibers (black asterisks). DVMs are out of focus in this picture. (D) Hemithoracic preparation of a wild-type pharate adult fly showing DLM muscle mass (black asterisks). Individual fibers cannot be clearly differentiated in pharate adult preparations. (E) Diagram of a hemisegment of an Antp-triple mutant pharate adult showing transformation in HT3. (F) Hemithoracic preparation of an Antp-triple mutant pharate adult showing DLMs (red asterisk) in HT3. Black asterisk indicates the DLM fibers in T2. All three hemithoracic preparations, shown in $\mathbf{B}, \mathbf{D}$, and $\mathbf{E}$, have been processed under identical conditions. Anterior: left; dorsal: top. Scale bar $=40 \mu \mathrm{m}$.

interactions between the ectoderm and mesoderm play an important role in regulating gene expression in adult myoblast populations. As muscle differentiation proceeds, the developing fiber interacts with the motor neurons, which innervate it. To examine what role the Hox identity of a motor neuron may have on its target muscles, we used the motor neuron GAL4 transgene, OK371-GAL4, to drive expression from a UAS-UbxRNAi transgene. Because Ubx is expressed in the T3 ectoderm, we examined the consequences on the muscles of this segment. Striking external deformities are seen in the legs of the T3 segment (Figure 7). Examination of the leg segments using polarized light show a reduced number of muscles in the femur (compare Figure 7B with 7A). The tibia in these animals shows a morphological deformity (compare Figure 7D with 7C), most probably arising as a result of a defect in musculature. Controls using en-GAL4 to drive the expression of
UAS-UbxRNAi gave expected phenotypes, transforming posterior haltere structures towards wing (data not shown). Again, when vg-GAL4 was used as a driver, haltere transformations to wing were seen. In a third control, when UAS-Ubx-RNAi is driven with elav-GAL4, sensory structures in the haltere are transformed towards wing. In all three cases no structures anterior to T2 are transformed. These results show that the Ubx-RNAi phenotypes are not artifacts.

A striking feature of Hox genes is the transformation of anterior structures to more posterior ones upon ectopic expression of 'posterior'-expressed genes. Thus, when $U b x$ is expressed in the T2 ectoderm, these structures are transformed towards T3 (Casares et al., 1996). In the light of our RNAi results, which suggest a requirement of $U b x$ in motor neurons in $\mathrm{T} 3$ for muscle development in that segment, we would expect that misexpression of $U b x$ in 

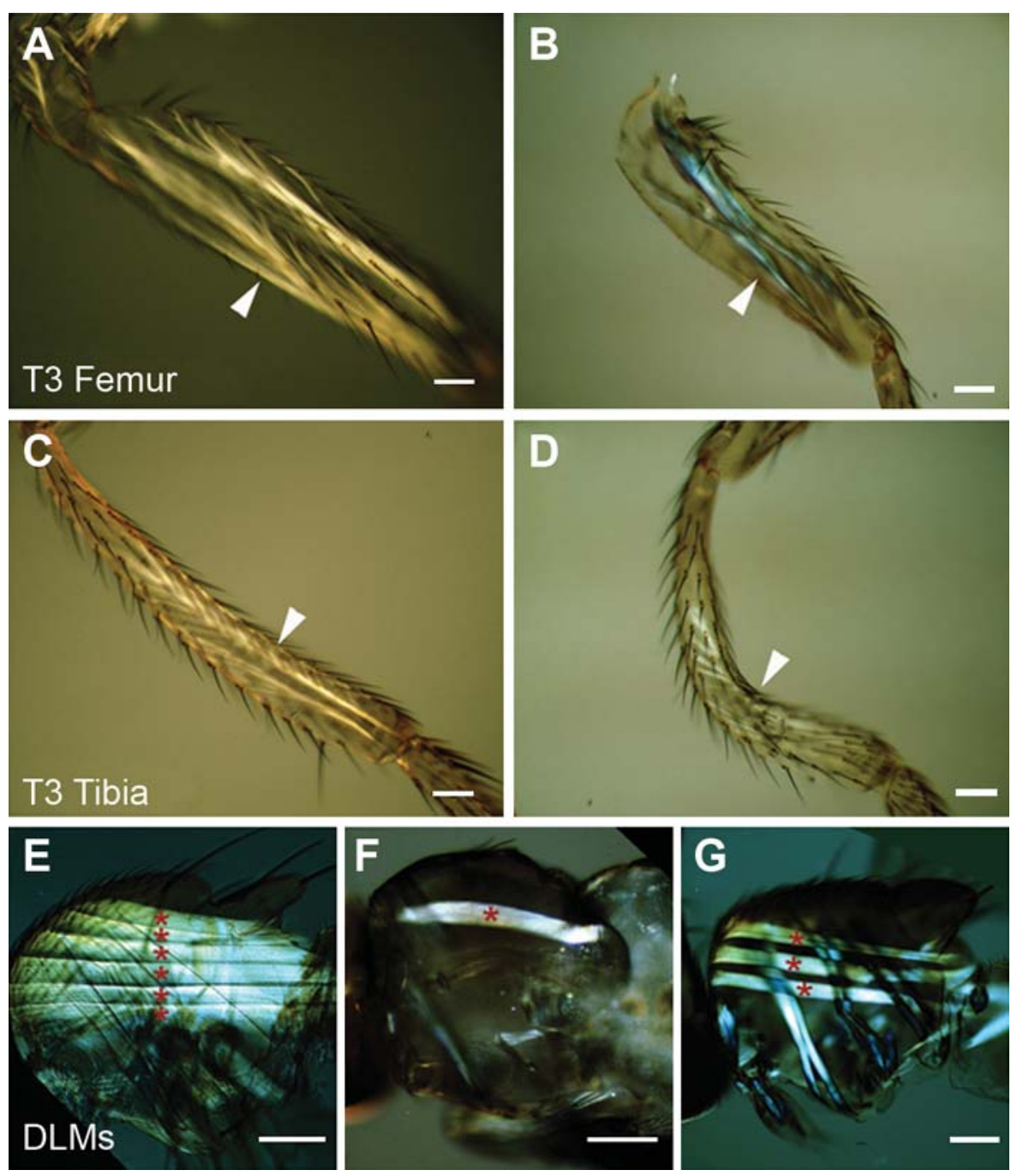

Figure 7. Alteration of levels of Ubx in motor neurons affects the development of the leg as well as the flight muscles. Leg (A-D) and flight $(\mathbf{E}-\mathbf{G})$ musculature imaged using polarized optics. Femur (A) and tibia (C) of OK371-GAL4 control animals showing welldeveloped musculature (arrowheads). Femur (B) and tibia (D) of animals where Ubx is down-regulated in motor neurons (UAS Dicer/+; OK371-Gal4/+; UAS-Ubx RNAi/+). Reduced muscles (arrowheads) lead to defects in leg morphology. (E) Thoracic preparations showing six well-developed dorsal longitudinal muscles (marked with asterisks) in adults of OK371-GAL4. (F) Misexpression of Ubx in motor neurons (OK371-Gal4/+; UAS-Ubx/+) leads to reduced number of DLMs (marked with asterisk). (G) Misexpression of Ubx in motor neurons that specifically innervate DLMs (P103.3-Gal4/+; UAS Ubx/+) leads to a reduction in the number of DLM fibers (marked with asterisk) in adult thorax. Scale bar $=100 \mu \mathrm{m}$ for $\mathbf{A}, \mathbf{B}, \mathbf{C}, \mathbf{D} ; 200 \mu \mathrm{m}$ for $\mathbf{E}, \mathbf{F}, \mathbf{G}$.

T2 motor neurons would result in an effect on T2 muscles development.

Using the OK371-GAL4 and the P103.3-GAL4 drivers, we next examined the effects of misexpressing Ubx in T2 motor neurons. Here too, striking effects are seen on the flight muscles of the dorsal thorax in lethal pharate pupae. With the OK371-GAL4 driver, almost no DLMs were present (Figure 7F), and with the P103.3-GAL4 driver, which marks motor neurons that innervate the DLMs, only three of the six DLMs were present in each hemisegment (Figure 7G compare to 7E).

We next examined the developmental provenance of these phenotypes. One possibility is that early events in myogenesis require the correct juxtaposition between the identities of nerve and muscle. If this were so, then an examination of flight muscle development upon $U b x$ misexpression in motor neurons may show an early phenotype. Indeed, this is the case (Figure 8). As early as $16 \mathrm{hAPF}$, when the splitting of the three larval templates to mature into the six DLMs are nearly complete in the wild-type (Figure 8A), experimental animals with misexpression of $U b x$ in the motor neurons show poor organization of the templates (Figure $8 \mathrm{~J})$. At $24 \mathrm{~h}$ APF, when the six DLM muscle fibers are well formed in the control animals (Figure 8D), the muscles of experimental animals are grossly disorganized (Figure $8 \mathrm{M}$ ). Twist-expressing myoblasts swarm around these poorly 


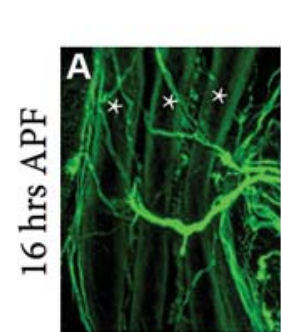

Wild-type
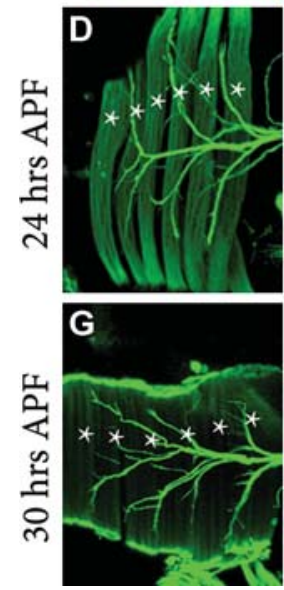
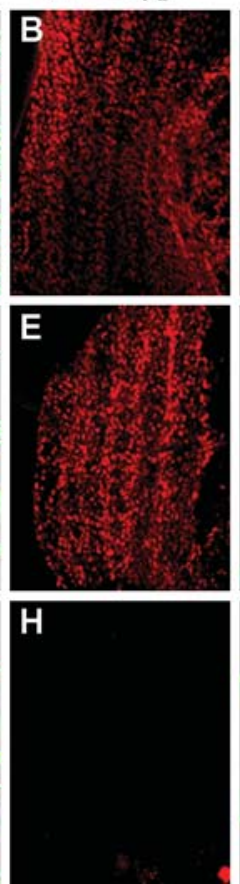
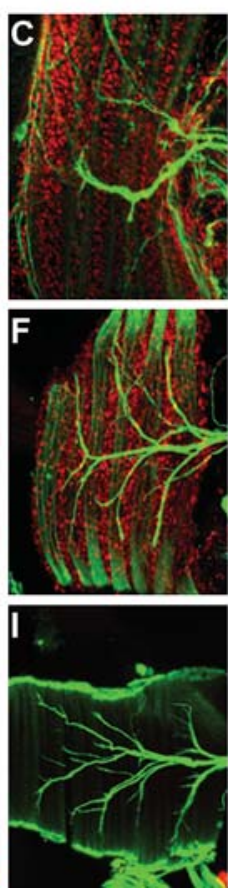
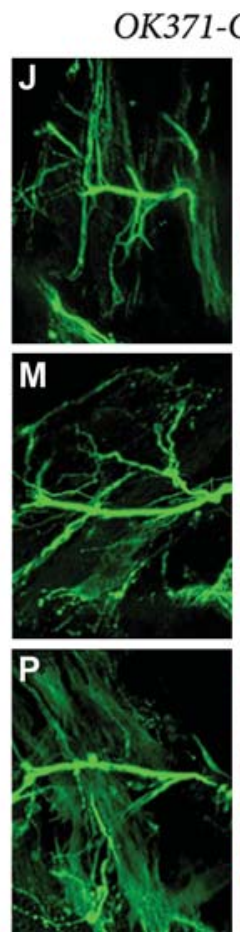
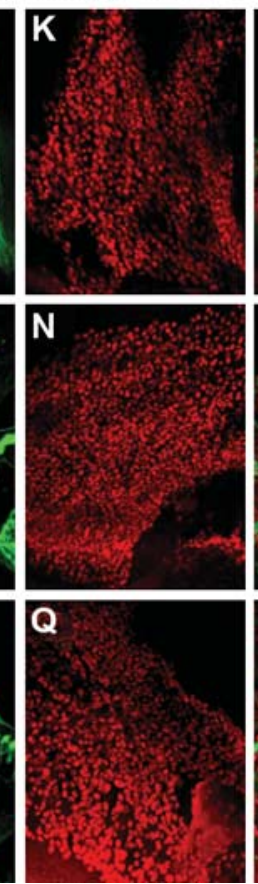
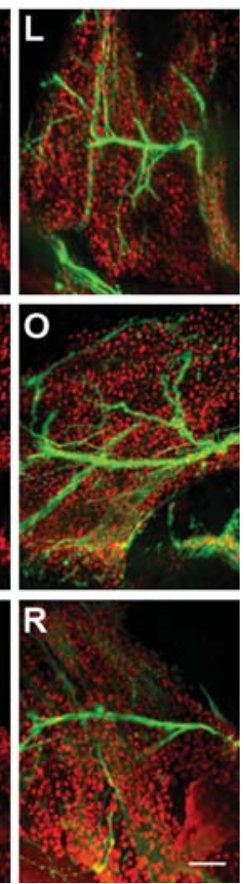

Figure 8. Misexpression of Ubx in T2 motor neurons results in early developmental defects in DLMs. Three key DLM developmental landmarks are represented. mAb 22 C10 staining is shown in green. 22C10 labels the microtubule-associated protein Futsch, and marks adult muscle founder cells (Dutta et al., 2004) and neurons (Hummel et al., 2000). The top row is at $16 \mathrm{~h}$ APF. At this stage, in the wildtype (A), the three larval templates (* in A) act as founders for the DLMs. Twist-expressing myoblasts (B, labeled in red) swarm over the templates, as can be seen in the merged picture $(\mathbf{C})$. These myoblasts will fuse with the templates, which will then split $(\mathbf{D}, 24 \mathrm{~h}$ APF) to form the six DLMs (* in D). At this stage, Twist-expressing myoblasts continue to surround the developing DLMs (E) and they are well organized around the developing fibers, as can be seen in the merged panel (F). By $30 \mathrm{~h}$ APF, the DLMs have matured $(*$ in $\mathbf{G})$. Twist expression is not seen any more $(\mathbf{H}$ and $\mathbf{I})$. In contrast to the wild-type, Ubx misexpression in motor neurons using the OK371Gal4 driver results in early developmental defects in DLM development. At 16 h APF (J), the templates are not oriented correctly as compared to the wild-type (A). In addition, Twist-expressing myoblasts, although present in large numbers at this stage (K), are not as well organized at this stage as compared with the wild-type (compare $\mathbf{K}$ with $\mathbf{B}$ ). L, a merged picture of $\mathbf{J}$ and $\mathbf{K}$, also brings out the poor $22 \mathrm{C} 10$ staining, suggestive of poor microtubule organization in the templates at this early stage when Ubx is misexpressed in motor neurons. As expected from the early defects, such animals at later stages ( $24 \mathrm{~h} \mathrm{APF)} \mathrm{show} \mathrm{severe} \mathrm{defects} \mathrm{in} \mathrm{DLM} \mathrm{development.}$ In contrast to the wild-type $(\mathbf{D}, \mathbf{E}, \mathbf{F})$, mutant animals $(\mathbf{M}, \mathbf{N}, \mathbf{O})$ show no significant fiber development (M), but Twist-expressing myoblasts ( $\mathbf{N}$ and $\mathbf{O}$ ) continue to envelop the region. At $30 \mathrm{~h}$ APF, misexpressed animals do not form DLM fibers (P), and myoblasts are still present in large numbers in the region and continue to express Twist ( $\mathbf{Q}$ and $\mathbf{R})$. This is in contrast to the wild-type where DLM fibers have formed $(\mathbf{G})$ and Twist expression is no longer seen $(\mathbf{H}, \mathbf{I})$. Scale bar $=50 \mu \mathrm{m}$.

developed muscles in an apparently random manner (Figure $8 \mathrm{~N}$ ), as opposed to the very well-organized arrangement seen in the wild-type (Figure 8E). Finally, at $30 \mathrm{~h}$ APF, when Twist expression has died down in control animals (Figure 8H) and the muscles are well organized (Figure 8G, I), animals with $U b x$ misexpression show persistent Twist expression (Figure 8Q). These myoblasts overlie the region where they would normally fuse to form fibers.

\section{DISCUSSION}

The formation of muscles is regulated by autonomous properties of the muscle-forming cells and also by inductive cues from the neighboring tissues-the epidermis and the innervating neurons (Buckingham \& Vincent, 2009;
Wigmore \& Evans, 2002; Ochi \& Westerfield, 2007; Dutta \& VijayRaghavan, 2005; Rochlin et al., 2009). Myogenic cells integrate inductive cues with their own intrinsic properties to form a segment-specific muscle pattern. The homeotic identities of inductive tissues, in addition to the autonomous homeotic identity of the muscle-forming cells, therefore, become crucial determinants of muscle pattern. We have used the IFMs in Drosophila as a model system to understand the relative roles of autonomous versus nonautonomous properties of Hox genes in governing muscle pattern.

Previous studies from our laboratory showed that several properties of the myoblasts, namely their spatial location on the disc and proliferation and migration patterns, were determined exclusively by the ectoderm (Fernandes et al., 1994; Roy \& VijayRaghavan, 1997). This 
was primarily deduced from studies with the triple-mutant four-winged mutants where a transformation in the Hox identity of the ectoderm, but not in the mesoderm, of T3 resulted in transformation of the aforesaid properties of the myoblasts to a T2 pattern. But these transformations were not sufficient to generate ectopic IFMs in HT3. The most probable explanation for this observation is that there are specific event(s) that require the correct Hox identity of the muscle-forming cells. Recent identification of two events critical for IFM formation - the segregation of IFM myoblasts into a Vg- and Ct-expressing subgroup and the function of founder cells in 'seeding' each myotube-allowed us to readdress this question. We set out to see if either or both of these events are disrupted in the triple mutants.

Sudarsan et al. (2001) have shown that IFM-forming myoblasts are segregated into a Vg-Ct-expressing subgroup. The maintenance of the expression of $\mathrm{Vg}$ in this group is shown to require Wingless signaling from the epidermis. It is possible, however, that the myoblasts are responsive to the $\mathrm{Wg}$ signal only if they have the correct homeotic address. If that is true, then myoblasts in the HT3 disc of triple-mutant larvae should not have a T2-like $\mathrm{Vg}$-Ct pattern. We find that $\mathrm{Vg}$-Ct expression in the myoblasts of HT3 disc of triple-mutant larvae was identical to that observed in wild-type $\mathrm{T} 2$. This showed that $\mathrm{Vg}$-Ct expression profile is guided entirely by ectodermal cues and rules out $\mathrm{Vg}-\mathrm{Ct}$ segregation as a cause for failure of IFM formation in triple-mutant flies.

Examination of the founder cells in the HT3 segment of triple-mutant pupae revealed significant results. We found that duf-lacZ-expressing founder cells for the DVMs were not observed in the HT3 of triple mutants. Larval templates-the 'founder analogues' of DLMswere also not observed in HT3 of the triple mutants. This would mean that even though the myoblasts were present in HT3 with the correct molecular identity in terms of $\mathrm{Vg}$ and $\mathrm{Ct}$ expression, they do not have the scaffolds to fuse to. Similar results, i.e., absence of founder cells, were observed in the HT3 of quad/triple-mutant flies, which have stronger ectodermal transformation than the triple mutants. However, decreasing the levels of Antp in the HT3 myoblasts of the triple mutant resulted in a significant number of pupae with DVM founders and larval templates in the transformed segment. The pharate adults of these mutants exhibited ectopic IFMs in their HT3 segment, indicating that the ectopic founders were capable of generating fibers and directing the myogenic process to completion. Although a more conclusive experiment for transforming the mesoderm would have been a complete removal of Antp exclusively from the mesoderm, our results suggest that Hox identity of the mesoderm is important for selection of IFM founders. Further support for this conclusion came from GAL4-UAS studies, where misexpression of Antp (or Ubx) in the T2 myoblasts (which normally do not express any Hox gene) abolished founder selection (D.D. and K.V.R., unpublished observations).

Although the absence of transformed IFMs in bithoraxcomplex mutants have been reported in several previous studies (Ferrus \& Kankel, 1981; Egger et al., 1990; Schneiderman 1993; Fernandes et al., 1994) and confirmed by our experiments, a study by Rivlin et al. (2001) have reported the presence of IFM-like fibers in the transformed T3 segment of individual $U b x$ alleles and different allelic combinations of $U b x$ (examined as hemizygotes over the deficiency $\operatorname{Df}(3 \mathrm{R}) \mathrm{P} 2)$. Their conclusion, that ectodermal cues are sufficient to generate the final IFM pattern, needs to be addressed in the context of our results. The fusion capability of the myoblasts is not dependent on their Hox identity (Roy \& VijayRaghavan, 1997). In case of the DLMs, the myoblasts can fuse to form fibers in the absence of larval templates (Farrell et al., 1996). It is possible therefore that ectodermal transformation of T3, achieved by mutant combinations in Rivlin et al., (2001) has influenced myoblasts to 'bypass' the founder-mediated mechanism and fuse with each other to form myotubes. Another possibility is that the muscles observed in their study are formed by late, ectopically chosen founders. This would suggest that the muscles seen are ectopic events and not IFMs. Even if this were the case, it is indeed puzzling that the authors see muscles in a situation where they, earlier (Egger et al, 1990, Schneiderman, 1993), and others including us (Ferrus \& Kankel, 1981; Fernandes et al., 1994; present study) have not been able to see. With the identification of founder cells and IFM markers, one can hope to resolve the reasons for the differences observed.

It remains to be seen the extent to which the dependence of founder cell selection on autonomous Hox identity is applicable for other sets of adult muscles. Interesting deviations are observed for the direct flight muscles (DFMs) and tergal depressor of the trochanter (jump muscle) present in the mesothorax. The formation of these muscles seems to be guided entirely by ectodermal signals. In triple-mutant and quad/triple-mutant flies, DFMs and jump muscles are present in the transformed segment (Fernandes et al., 1994; Schneiderman et al., 1993). Whether these ectopic muscles possess the wild-type molecular characteristics has not been examined. Expression of duf-lacZ in wildtype mature DFMs and jump muscles (D.D. and K.V.R., unpublished observations) conforms to their development via a founder-dependent mechanism. It would be interesting to see whether these ectopic DFMs and jump muscles develop by a founder-mediated mechanism.

Our results on the consequences on muscle of downregulating or misexpressing Ubx in motor neurons demonstrates a new preparation for studying the role of the nervous system in myogenesis. Studies in mouse have demonstrated the requirements of motor inputs for muscle maintenance, an interaction whose underlying mechanism is poorly understood (Finol et al., 1981). Motor neuron 
function has also been shown to influence the isoform choice of contractile protein gene expression (Harris et al., 1989). Here too, the mechanistic basis of these influences of the nerve on muscle is not well understood. A genetically tractable system where the role of the nervous system in muscle development and maintenance can be studied is therefore valuable. A preparation where this question can be addressed effectively is the muscle of Lawrence (MOL) in the 5th abdominal segment of the male adult fly (Gailey et al., 1991). The sex-specific and segment-specific presence of this muscle is dictated by the nervous system (Gailey et al., 1991). We now show, by using motor neuron drivers and RNAi tools, that motor neurons could have wide roles in muscle morphogenesis or maintenance. Several tantalizing and new features emerge from the results of Ubx-RNAi and Ubx-ectopic expression in motor neurons. The loss-of function experiments suggest an important role of the nervous system in muscle development. Because the T3 muscles are poorly investigated developmentally as compared to the flight muscles, the misexpression study is valuable in corroborating a role for the nervous system in myogenesis. The early developmental defects suggest that interactions between developing motor neurons and their targets could have an important role in fiber maturation.

Although experiments with the male-specific abdominal muscle in A5 had shown a role for the nervous system in muscle development, other experiments in the embryo that showed that muscle development took place independent of innervation (Broadie \& Bate, 1993) suggested that the adult 'muscle of Lawrence' role of the nervous system may have been an exception. Here we show that down-regulation of Ubx, using a motor neuron GAL4 driver, leads to striking defects in the musculature of the T3 legs. The domain of function of Ubx in the ectoderm is from T3 posterior and, therefore, the T3 effect is consistent with this. Similarly, misexpression of Ubx in $\mathrm{T} 2$ motor neurons also results in strong early developmental effects on the IFMs.

Our results point to a more general role for the nervous system in muscle patterning. What could be the mechanistic underpinning of this interaction? Although the possibilities range from effects on muscle-fiber gene expression (Harris et al., 1989) to myoblast proliferation, as has been suggested from ablation experiments (Fernandes \& Keshishian, 2005), these remain speculative. However, our genetic preparation allows the dissection of these interactions.

Our present results, taken together with results of earlier studies (Greig \& Akam, 1993; Fernandes et al., 1994; Roy et al., 1997; Roy \& VijayRaghavan, 1997), give a picture of how concerted activity of Hox genes during different aspects of indirect flight myogenesis makes the outcome of that process segment specific. Like many other molecular functions that have been shown to be conserved between vertebrates and lower animals, we suggest that much of what has been gathered from experiments on the functions of homeotic genes in diverse aspects of myogenesis in Drosophila will reflect the manner in which vertebrate Hox genes would function during pattern formation in the somatic mesoderm of vertebrates.

\section{ACKNOWLEDGMENTS}

The authors would like to thank Sean Carroll, Danny Brower, Siegfried Roth, and Daniel Kiehart for antibodies. The support of the Central Imaging and Flow Facility of NCBS is gratefully acknowledged. This article has been written in honour of Erich Buchner, who has inspired many collaborators and colleagues hoping to follow his example and his joy of doing science. K.V.R. and V.R. would particularly like to express their gratitude to Erich for many fruitful interactions. V.R. was a postdoctoral fellow in Erich Buchner's group at the Max-Planck Institut für biologische Kybernetik, Tübingen, Germany.

Declaration of interest: This work was supported by the Department of Biotechnology (DBT), The Indo-French Centre for Advanced Scientific Research (CEFIPRA/ IFCPAR), and the National Centre for Biological Sciences (NCBS), Tata Institute of Fundamental Research, to K.V.R. and a Kanwal Rekhi Fellowship to D.D.

\section{REFERENCES}

Bate, M., Rushton, E., \& Currie, D. A. (1991). Cells with persistent twist expression are the embryonic precursors of adult muscles in Drosophila. Development, 113, 79-89.

Bour, B. A., Chakravarti, M., West, J. M., \& Abmayr, S. M. (2000). Drosophila SNS, a member of the immunoglobulin superfamily that is essential for myoblast fusion. Genes Dev, 14, 1498-1511.

Brierley, D. J., Blanc, E., Reddy, O. V., VijayRaghavan, K., \& Williams, D. W. (2009). Dendritic targeting in the leg neuropil of Drosophila: The role of midline signalling molecules in generating a myotopic map. PLoS Biol, 7, e1000199.

Broadie, K., \& Bate, M. (1993). Muscle development is independent of innervation during Drosophila embryogenesis. Development, 119, 533-543.

Buckingham, M., \& Vincent, S. D. (2009). Distinct and dynamic myogenic populations in the vertebrate embryo. Curr Opin Genet Dev, 19, 444-453.

Casares, F., Calleja, M., \& Sánchez-Herrero, E. (1996). Functional similarity in appendage specification by the Ultrabithorax and abdominal-A Drosophila HOX genes. EMBO J, 15, 3934-3942.

Consoulas, C., Restifo, L. L., \& Levine, R. B. (2002). Dendritic remodeling and growth of motoneurons during metamorphosis of Drosophila melanogaster. J Neurosci, 22, 4906-4917.

Currie, D. A., \& Bate, M. (1991). The development of adult abdominal muscles in Drosophila: Myoblasts express twist and are associated with nerves. Development, 113, 91-102. 
DeSimone, S., Coelho, C., Roy, S., VijayRaghavan, K., \& White, K. (1995). ERECT WING, the Drosophila member of a family of DNA binding proteins is required in imaginal myoblasts for flight muscle development. Development, 121, 31-39.

Dutta, D.,Anant, S., Ruiz-Gomez, M., Bate, M., \& VijayRaghavan, K. (2004). Founder myoblasts and fibre number during adult myogenesis in Drosophila. Development, 131, 3761-3772.

Dutta, D., Shaw, S., Maqbool, T., Pandya, H., \& VijayRaghavan, K. (2005). Drosophila Heartless acts with Heartbroken/Dof in muscle founder differentiation. PLoS Biol, 3, e337.

Dutta, D., \& VijayRaghavan, K. (2005). Metamorphosis and the formation of the adult musculature. In H. Sink (Ed.), Muscle development in Drosophila (pp. 125-142). Georgetown, TX: Landes Biosciences.

Egger, M. D., Harris, S., Peng, B., Schneiderman, A. M., \& Wyman, R. J. (1990). Morphometric analysis of thoracic muscles in wild-type and in bithorax Drosophila. Anat Rec, 226, 373-382.

Farrell, E. R., Fernandes, J., \& Keshishian, H. (1996). Muscle organizers in Drosophila: The role of persistent larval fibres in adult flight muscle development. Dev Biol, 176, 220-229.

Fernandes, J., Bate, M., \& VijayRaghavan, K. (1991). Development of the indirect flight muscles of Drosophila. Development, 113, 67-77.

Fernandes, J., Celniker, S. E., Lewis, E. B., \& VijayRaghavan, K. (1994). Muscle development in the four-winged Drosophila and the role of the Ultrabithorax gene. Curr Biol, 4, 957-964.

Fernandes, J. J., Celniker, S. E., \& VijayRaghavan, K. (1996). Development of the indirect flight muscle attachment sites in Drosophila: Role of the PS integrins and the stripe gene. Dev Biol, 176, 166-184.

Fernandes, J., \& Keshishian, H. (1996). Patterning the dorsal longitudinal flight muscles (DLM) of Drosophila: Insights from the ablation of larval scaffolds. Development, 122, 3755-3763.

Fernandes, J. J., \& Keshishian, H. (2005). Motoneurons regulate myoblast proliferation and patterning in Drosophila. Dev Biol, 277, 493-505.

Fernandes, J., \& VijayRaghavan, K. (1993). The development of indirect flight muscle innervation in Drosophila melanogaster. Development, 118, 215-227.

Ferrus, A., \& Kankel, D. R. (1981). Cell lineage relationships in Drosophila melanogaster: The relationships of cuticular to internal tissues. Dev Biol, 85, 485-504.

Finol, H. J., Lewis, D. M., \& Owens, R. (1981). The effects of denervation on contractile properties or rat skeletal muscle. J Physiol, 319, 81-92.

Gailey, D. A., Taylor, B. J., \& Hall, J. C. (1991). Elements of the fruitless locus regulate development of the muscle of Lawrence, a male-specific structure in the abdomen of Drosophila melanogaster adults. Development, 113, 879-890.

Ghazi, A., Anant, S., \& VijayRaghavan, K. (2000). Apterous mediates development of direct flight muscles autonomously and indirect flight muscles through epidermal cues. Development, 127, 5309-5318.

Greig, S., \& Akam, M. (1993). Homeotic genes autonomously specify one aspect of pattern in the Drosophila mesoderm. Nature, 362, 630-632.
Harris, A. J., Fitzsimons, R. B., \& McEwan, J. C. (1989). Neural control of the sequence of expression of myosin heavy chain isoforms in foetal mammalian muscles. Development, 107, 751-769.

Hummel, T., Krukkert, K., Roos, J., Davis, G., \& Klämbt, C. (2000). Drosophila Futsch/22C10 is a MAP1B-like protein required for dendritic and axonal development. Neuron, 26, 357-370.

Lawrence P. A. (1982). Cell lineage of the thoracic muscles of Drosophila. Cell, 29, 493-503.

Lewis, E. B. (1978). A gene complex controlling segmentation in Drosophila. Nature, 276, 565-570.

Lewis. E. B. (1980). Report of new mutants: Drosophila melanogaster. Drosophila Inf Serv, 55, 207-208.

Mahr, A., \& Aberle, H. (2006). The expression pattern of the Drosophila vesicular glutamate transporter: A marker protein for motoneurons and glutamatergic centers in the brain. Gene Expr Patterns, 6, 299-309.

Nose, A., Isshiki, T., \& Takeichi, M. (1998). Regional specification of muscle progenitors in Drosophila: The role of the msh homeobox gene. Development, 125, 215-223.

-Ochi, H., \& Westerfield, M. (2007). Signaling networks that regulate muscle development: Lessons from zebrafish. Dev Growth Differ, 49, 1-11.

Rivlin, P. K., Gong, A., Schneiderman, A. M., \& Booker, R. (2001). The role of Ultrabithorax in the patterning of adult thoracic muscles in Drosophila melanogaster. Dev Genes Evol., 211, 55-66.

Rochlin, K., Yu, S., Roy, S., \& Baylies, M. K. (2010). Myoblast fusion: When it takes more to make one. Dev Biol, 34, 66-83.

Roy, S., Shashidhara, L. S., \& VijayRaghavan K. (1997). Muscles in the Drosophila second thoracic segment are patterned independently of autonomous homeotic gene function. Curr Biol, 7, 222-227.

Roy, S., \& VijayRaghavan, K. (1997). Homeotic genes and the regulation of myoblast migration, fusion, and fibre-specific gene expression during adult myogenesis in Drosophila. Development, 124, 3333-3341.

Roy, S., \& VijayRaghvan, K. (1999). Muscle pattern diversification in Drosophila: The story of imaginal myogenesis. Bioessays, 21, 486-498.

Ruiz-Gomez, M., Coutts, N., Price, A., Taylor, M. V., \& Bate, M. (2000). Drosophila dumbfounded: A myoblast attractant essential for fusion. Cell, 102, 189-198.

Schneiderman, A. M., Tao, M. L., \& Wyman, R. J. (1993). Duplication of the escape-response neural pathway by mutation of the bithorax-complex. Dev Biol, 157, 455-473.

Sudarsan, V. P., Anant, S., Guptan, P., Skaer, H., \& VijayRaghavan, K. (2001). Myoblast diversification and ectodermal signalling in Drosophila. Dev Cell, 1, 829-839.

Waddington, C. H., \& Robertson, E. (1966). Selection for developmental canalisation. Genet Res, 7, 303-312.

Wigmore, P. M., \& Evans, D. J. (2002). Molecular and cellular mechanisms involved in the generation of fibber diversity during myogenesis. Int Rev Cytol, 216, 175-232. 